\title{
RFID BASED EMBEDDED SECURITY SYSTEM
}

\author{
Shamika Likhite ${ }^{1^{*}}$, Nishikant Lambat ${ }^{2 *}$, Vinit Kavalekar ${ }^{3^{*}}$, Tarun Kumar Yadav $^{4 *}$, Pranav Pati ${ }^{1^{*}}$, \\ Harshad Zade ${ }^{6}$, Uddesh Tople ${ }^{7}$
}

Visvesvaraya National Institute of Technology, South Ambazari Road, Nagpur, Maharashtra, India 440010

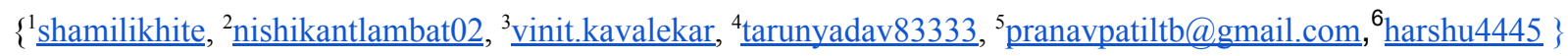

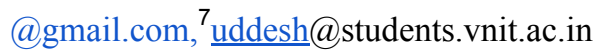

\begin{abstract}
The paper presents the design and development of the RFID based embedded the GSM module as the processors and the LCD as the display. This system aims at delivering a text message notification to the owner on an employee's entry. In addition, the system also sends a video footage recording to the owner on a guest entry along with text notification. The background of the study, the design approaches for this system and the preliminary evaluation of the prototype are presented in this paper. The evaluation results indicate that the system is useful and easy to use for security purposes.
\end{abstract}

Keywords: RFID reader, RFID tag, GSM module, LCD display, Arduino Mega 2560, RC522, Raspberry pi 0 w, Raspberry Pi camera module, SPI, EEPROM, USBasp, UART.

\section{INTRODUCTION}

Nowadays many security and anti-theft options are available. But these options are pretty expensive. So there is a need for a Security system that is quite economical and easy to use. RFID, which stands for Radio Frequency identification is one such option available used for retrieving from or storing data on the RFID tag without any physical contact. The RFID based embedded security system comprises of the RFID tags, RFID reader(RC522), GSM module SIM 900A, Raspberry Pi camera module, 12V solenoid lock. The RFID tags were passively activated, where the RFID reader reads the tag when scanned. This system uses an Arduino Mega 2560 module of microcontroller which interfaces with other devices like the GSM module SIM900A, RFID reader (RC522). Raspberry pi $0 \mathrm{~W}$ model is used to interface the Raspberry pi camera module. When an employee has to access the security box the employee will scan his RFID tag to open the solenoid door lock. After the card is scanned the admin will get a text message notification of the name and the tag number of the card scanned. The door lock system is designed using a solenoid door lock which is controlled using a relay. When an unauthorized person wants to open the door lock he/she is unable to open the door without the RFID tag/a fake RFID tag. There may be a possibility that a visitor wants to access the security system. For this provision of a guest card entry is made. When a guest named RFID tag is scanned a text notification is sent to the admin along with the video footage recording of about 10 seconds is recorded which can be accessed using the FTP server on the local network. The system and the device should be connected to the same server to access the video recording. The card name of the card scanned by the RFID reader is displayed on the $16 \times 2$ LCD display. The LCD display gives a default message to scan the tag otherwise.

The SIM 900A of the GSM module uses a UART serial communication protocol for interfacing with the Arduino Mega 2560 microcontroller. Similarly, the RFID Reader uses SPI serial communication protocol for interfacing with the microcontroller. The connection between the RFID tag and the RFID reader is through the 
electromagnetic field produced due to mutual inductance. The reader's antenna is used to transmit Radio frequency $(\mathrm{RF})$.

\section{RFID TECHNOLOGY}

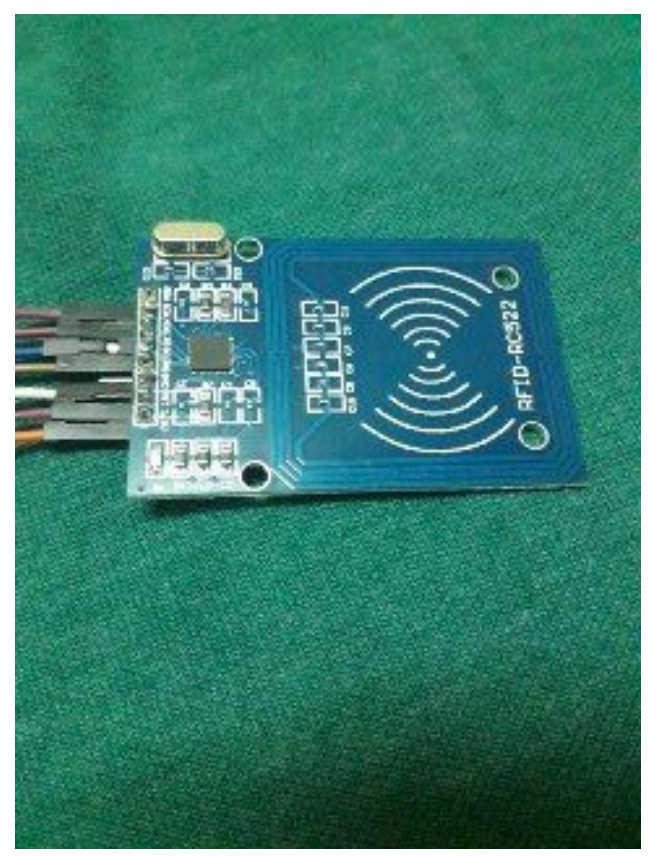

\section{Fig.1. RFID Reader (Surface with antennas)}

RFID, "Radio Frequency Identification" is the wireless technology based on electromagnetic waves. It consists of a Reader and a Tag. Tag has a microchip that contains a unique 8 bytes/characters. The main principle of the working of the system is powering the RFID tag and the data transmission between RFID Reader and RFID Tag. As shown in fig. RFID Reader consists of an antenna that transmits (as well as receives) the electromagnetic waves which energizes the winding in RFID tag. This activates the microchip present in the Tag. Now winding in RFID Reader acts as the primary of the transformer and the winding in RFID tag acts as a secondary of the transformer due to mutual inductance between two winding very close to each other.

As per the working principle of a transformer, the current in primary winding varies as per the current in the secondary winding. If the secondary has zero current primary current remains as it is (we call it no-load current) but this primary current changes as the secondary current changes according to secondary conditions.

Following the above transformer principle, when Tag is encountered with electromagnetic waves sone energy is executed for the powering of the microchip and the other part flows as secondary current. Microchip contains a unique RFI ID which is a code in binary form which switches ON/OFF the secondary circuitry for 1 and 0 respectively. This results in changing primary current i.e. current in RFID reader which eventually transfers the Data stored in the Tag-microchip to the Reader and to the system. This notifies that the whole system is a mini transformer. 
Depending upon the type of Reader and the Tag, the system is classified into various types. Depending upon the frequency usage we get various classifications of RFID systems. Available frequencies include low frequency ( $125 \mathrm{kHz})$, high-frequency (13.56 MHz) and ultra-high-frequency or UHF (860-960 MHz) [1].

\section{SYSTEM DEVELOPMENT}

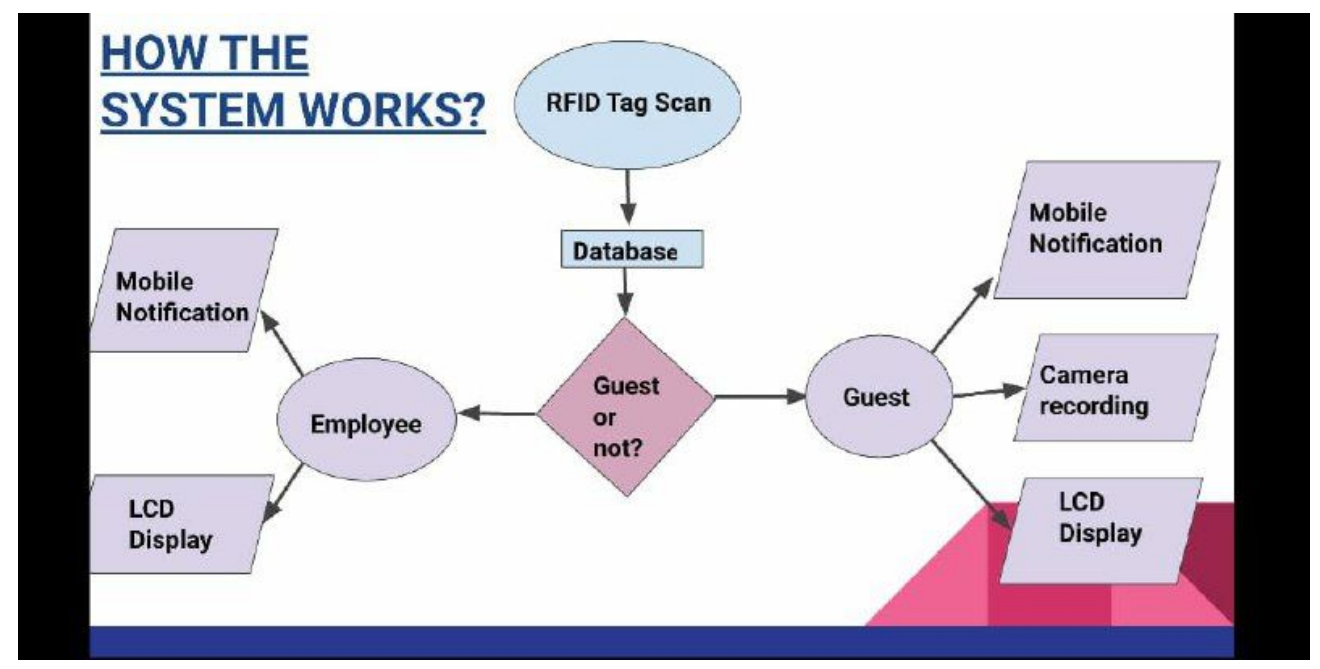

\section{Fig.2.Block diagram of the Security system.}

Figure 1 gives an idea of how the RFID security system works.

3.1 Software: We developed the prototype using Atmel studio 7.0 using $\mathrm{C}++$ programming and Simulated on proteus PCB simulator. This language is chosen due to the simplicity in coding for interfacing with the RFID hardware.

3.2 User Interface: There are two main types of user interface of the RFID system. One is for the admin when an employee or the guest scans the tag and the other is for the employee and the guest who scans the tag by displaying the status of the tag on the LCD display.

3.3 Hardware: As hardware, we have interfaced with RC522 as an RFID Reader and a passive RFID tag(s) and a SIM 900a GSM module for sending a text message to the admin with ATMega 2560 microcontroller. An Rpi camera module was interfaced using Raspberry pi $0 \mathrm{w}$.

To display the state of the security system an LCD 16x2 display was used. The system consists of two main parts. The first part controls scanning and computation and the second part controls the actuator (Solenoid lock), Camera recording and message conveying system to notify the admin.

3.3.1 Microcontroller: Microcontroller used here is Atmega2560. It is an 8-bit microcontroller with 54 general purpose input output pins (of which 14 can be used as PWM outputs), 16 analog inputs, 4 UARTs (hardware serial ports). 


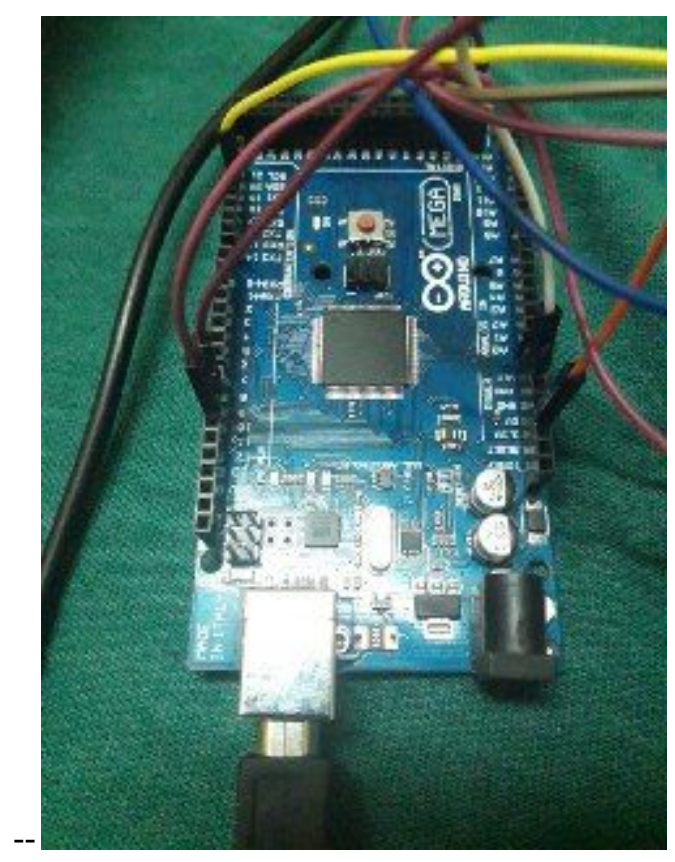

Fig.1.Outlook of Arduino Mega

Figure 1. Shows the display look of the Arduino mega. Arduino Mega uses Atmega 2560 microcontroller for operations..

3.3.2 GSM: Global System for Mobile Communications (GSM) is a famous technology in the world. Throughout the evolution of cellular telecommunications, various systems have been developed without the benefit of standardized specifications[2]. This presented many problems directly related to compatibility, especially with the development of digital radio technology[2]. The GSM standard is intended to address these problems. SM provides recommendations, not requirements[2]. The GSM specifications define the functions and interface requirements in detail but do not address the hardware[2]. The reason for this is to limit the designers as little as possible but still to make it possible for the operators to buy equipment from different suppliers[2]. The GSM network is divided into three major systems: the switching system (SS), the base station system (BSS), and the operation and support system (OSS)[9]. The mobile device connects to the network via hardware. The subscriber identity module (SIM) card provides the network with identifying information about the mobile user[9].

3.3.3 Radiofrequency identification (RFID) tag and reader: RC522 is the RFID reader module with the SPI interface. RFID reader modules are available in UART as well as the SPI interface for any microcontroller. Due to the internal structure of these protocols, the transmission speed for these modules is different and with SPI supported module with higher speed. RC522 module has a blue colored body with total 8 pins out of which one is for interrupt which was not used here. Other pins include MOSI, MISO, SCK, SDA, GND, VCC (3V), RST. Each and every pin has a specified function. RC522 Reader is a highly integrated Reader/Writer IC for contactless communication at $13.56 \mathrm{MHz}$. It is applicable for only passive tags for a range of about $3 \mathrm{~cm}$ from the antenna surface. It can detect tag in free-falling cases falling from a height of about $30 \mathrm{~cm}$. Tags can be recognized/scanned even if kept inside a wallet or a case.

3.3.4 Camera: Raspberry Pi camera module was used to record high -definition videos as well as still photographs. The Raspberry Pi Camera v2 is the new official camera board released by the Raspberry Pi Foundation. The 
Raspberry Pi Camera Module v2 is a high quality 8 megapixel Sony IMX219 image sensor custom designed add-on board for Raspberry Pi, featuring a fixed focus lens.

3.3.5 Raspberry Pi: This is the slimmest, most pared down Raspberry Pi to date. It's kind of like the little cousin to the Pi 3 - with just a micro SD card slot, a mini HDMI port, two micro USB ports (one for power, one for USB), and $512 \mathrm{MB}$ of RAM. It has a single-core $1 \mathrm{GHz}$ processor chip, similar to the $\mathrm{Pi} \mathrm{A}+$ and $\mathrm{B}+$.

3.3.6 USBasp Programmer: USBasp is a USB in-circuit programmer for Atmel AVR controllers. It simply consists of an ATMega88 or an ATMega8 and a couple of passive components. The programmer uses a firmware-only USB driver, no special USB controller is needed.This was very helpful to program the backbone of the security system i.e. Microcontroller present Atmega 25600when it is programmed in Arduino ide the serial communication in microcontroller as a master and USB asp as a slave is SPI where it is serial peripheral interface on the other hand when microcontroller is high all the erasable data is cleared whereas the code in the EEPROM remains unchanged. Thus no need to reprogram the bootloader.

3.3.7 LCD Display: LCD Pin Description: LCD is the common interface between software, hardware, and user. LCD pin configuration is as follows VCC, VSS, VEE VCC \& VSS provide $+5 \mathrm{~V} \&$ ground respectively, VEE is used for adjusting LCD contrast. RS ( Register Select). If $\mathrm{RS}=0$, the instruction command code register is selected, allowing the user to send a command such as clear display, cursor at home, etc. If RS $=1$, data to be displayed on $\mathrm{LCD}$ [7]. $\mathrm{R} / \mathrm{W}$ (Read/Write). If $\mathrm{R} / \mathrm{W}=1$, Read operation. If $\mathrm{R} / \mathrm{W}=0$, Write operation. The $\mathrm{LCD}$, to latch information presented to its data pins uses the Enable pin. When data is supplied to data pins, a high - to - low pulse must be applied to this pin in order to latch data present at the data pins. When D7 $=1$, the LCD is used for internal operations and will not accept any new information. When D7 $=0$, the LCD is ready to receive new data. LCD used here is $16 \times 2[7]$.

\section{CONCLUSION, SHORTCOMINGS AND FURTHER SCOPE}

In this paper, we tried to implement a cost-effective and easier way of security. Coding for the entire system was done on the register level without using complex libraries which resulted in both time and space-efficient security systems which makes it robust for any platform. The guest entry was monitored by the video footage recorded by the Raspberry Pi camera module. This added a scope of security. The RFID security system tag cannot be imprinted unless one knows the entire 8-byte tag ID. The LCD displays only the name of the tag associated with that tag number. For further improvements, the system can be added with a buzzer or an alarm that rings when a certain number of false attempts are made while scanning an invalid tag. This will further gear up the security of the things inside the RFID box.

\section{REFERENCES}

1. Nazleeni S. Haron, Nur S. Saleem, Mohd H. Hasan, Mazeyanti M. Ariffin and Izzatdin A. Aziz, A RFID-based Campus Context-Aware Notification System (March,2010).

2. Shital Y. Gaikwad and Maheshwari V. C.Security System for Car using RFID, Thumb Impression, Steering wheel Lock Based on ARM 7

3. P Raaj Vignesh, M Vasantha Kumar, Mary Joseph, Development of fishermen border alert and speed reduction system using RFID with GSM alert.

4. RFID Journal, http://www.rfidjournal.com.2009 
5. Aim Global, “Technologies: RFID/What is RFID?” Aim Globa http://www.aimglobal.org/technologies/rfid/what_is_rfid.asp.2009

6. Interfacing SIM900A GSM Modem With Arduino, https://www.instructables.com/id/Interfacing-SIM900A-GSM-Modem-with-Arduino

7. Interfacing LCD with Atmega32 Microcontroller using Atmel Studio, https://electrosome.com/interfacing-lcd-atmega32-microcontroller-atmel-studio/

8. Serial communication, http://www.circuitbasics.com/

9. GSM (Global System forMobilecommunication),https://searchmobilecomputing.techtarget.com/definition/GSM 